\title{
Philosophiques
}

\section{D’une réduction phénoménologique pratique. Scheler, Heidegger et l'appel de la conscience}

\section{Paul Slama}

Volume 45, numéro 1, printemps 2018

URI : https://id.erudit.org/iderudit/1048619ar

DOI : https://doi.org/10.7202/1048619ar

Aller au sommaire du numéro

Éditeur(s)

Société de philosophie du Québec

ISSN

0316-2923 (imprimé)

1492-1391 (numérique)

Découvrir la revue

Citer cet article

Slama, P. (2018). D’une réduction phénoménologique pratique. Scheler, Heidegger et l'appel de la conscience. Philosophiques, 45(1), 159-180. https://doi.org/10.7202/1048619ar
Résumé de l'article

Dans cet article, je souhaite comprendre comment Max Scheler et Martin Heidegger comprennent les normes sociales pour nos perceptions et nos pratiques quotidiennes, et comment ils décrivent la nécessité d'un dépassement de cette normativité au profit d'une autre, plus " personnelle " ou encore plus " authentique ". Je montre que c'est un appel de la conscience qui rompt la normativité sociale, et qui engage l'être humain à ne plus agir mécaniquement, mais à vivre librement. Cette rupture avec la normativité sociale est une réduction phénoménologique que nous appelons « réduction éthique ». Tout l'enjeu de l'article est de montrer qu'une telle réduction repose en fait sur les normativités sociales en tant qu'elles sont le critère de l'appel, le milieu où un tel appel est possible. De ce point de vue, le sujet réflexif demeure un sujet social. 


\title{
D'une réduction phénoménologique pratique. Scheler, Heidegger et l'appel de la conscience'
}

\author{
PAUL SLAMA \\ Université Paris-Sorbonne
}

\begin{abstract}
RÉSUMÉ. - Dans cet article, je souhaite comprendre comment Max Scheler et Martin Heidegger comprennent les normes sociales pour nos perceptions et nos pratiques quotidiennes, et comment ils décrivent la nécessité d'un dépassement de cette normativité au profit d'une autre, plus «personnelle» ou encore plus «authentique». Je montre que c'est un appel de la conscience qui rompt la normativité sociale, et qui engage l'être humain à ne plus agir mécaniquement, mais à vivre librement. Cette rupture avec la normativité sociale est une réduction phénoménologique que nous appelons «réduction éthique». Tout l'enjeu de l'article est de montrer qu'une telle réduction repose en fait sur les normativités sociales en tant qu'elles sont le critère de l'appel, le milieu où un tel appel est possible. De ce point de vue, le sujet réflexif demeure un sujet social.
\end{abstract}

\begin{abstract}
In this article, I want to understand how Max Scheler and Martin Heidegger understand the social norms for our perceptions and our daily practices, and how they describe the need to go beyond this normativity in favor of another, more "personal" or even more "authentic". I defend that it is a call of consciousness that breaks the social normativity, and which commits the human being to no longer act mechanically, but to live freely. This break with social normativity is a phenomenological reduction that I call "ethical reduction". The whole point of the article is to show that such a reduction is in fact based on social normativities as they are the criterion of the call, the environment in which such a call is possible. From this point of view, the reflective subject remains a social subject.
\end{abstract}

Comme quelques commentateurs y ont insisté, il y a bien à l'œuvre, dans le traité Être et temps (I927), la pratique d'une réduction phénoménologique². Pour résumer la teneur de cette réduction, il s'agit pour Heidegger de briser le bavardage inauthentique pris dans la quotidienneté, en somme briser la normativité sociale habituelle, pour que le Dasein puisse revenir à lui-même.

1. Je remercie les deux lecteurs anonymes pour leurs précieuses suggestions et corrections qui m'ont permis de réviser substantiellement l'article en vue de sa publication.

2. Jean-François Courtine, Heidegger et la phénoménologie, Paris, Vrin, I992, p. 229. Didier Franck avait pour sa part, dans Heidegger et le problème de l'espace, Paris, Minuit, I986, p. 73-74, déjà reconnu le lien entre réduction et l' "angoisse »: «L'angoisse assure dans l'analytique existentiale une fonction méthodique cardinale, analogue à celle de la réduction transcendantale pour l'analyse intentionnelle de la subjectivité: elle révèle le Dasein à luimême dans son être-au-monde vers la mort. » J.-F. Courtine, pour sa part, présente son hypothèse en ces termes (op. cit., p. 234): "Dans SuZ, c'est l'analyse de l'angoisse qui constitue comme la "répétition" de la problématique husserlienne de l'épochè et de la réduction phénoménologique-transcendantale.»

PHILOSOPHIQUES 45/1 — Printemps 2018, p. 159-180 
L'être humain se détourne alors du simple usage des choses dans le contexte social où il se trouve pour accéder à un niveau plus fondamental où il ne dépend plus de ce contexte et où il peut d'ailleurs en entreprendre l'analyse. En d'autres termes, il s'agit pour Heidegger d'attester la possibilité pour l'être humain de passer d'un contexte où la normativité est purement sociale (ce qu'il appelle la tyrannie du "on») à un plan où le Dasein est avec luimême, où il peut se retrouver, se connaître et ainsi être plus authentiquement au monde. Réduction, c'est-à-dire reconduction, du plan quotidien, socialement normé (ce n'est pas «moi» qui pratique le marteau, mais «on» le pratique, et chacun le pratique de la même façon et en vue des mêmes buts) à un plan plus originaire, qui est un plan phénoménologiquement attestable, descriptible.

Cette pratique post-husserlienne de la réduction phénoménologique n'est pas seulement heideggérienne. Elle est aussi le fait de Max Scheler, qui appartient comme Heidegger à la première génération des disciples de Husserl, et qui a très vite transformé les thématiques fondamentales de la phénoménologie en thématiques pratiques. L'ambition de notre étude n'est pas de faire de Scheler une simple influence pour Heidegger; on souhaite bien plutôt aborder l'un et l'autre penseurs à partir de la communauté de leurs approches: si l'un et l'autre sont des penseurs de la praxis humaine dans le monde, si l'un et l'autre ont pensé les phénomènes comme des signes qui renvoient à un usage possible, et s'ils ont décrit l'intentionnalité en termes de pratique, l'un et l'autre ont aussi pensé la possibilité éthique de sortir d'un tel monde au moyen d'une réduction phénoménologique, pour accéder à une dimension plus fondamentale de soi-même. C'est du contrat phénoménologique qu'il s'agit alors (si l'on parle, et seulement, de la phénoménologie transcendantale inventée par Husserl), c'est-à-dire la possibilité de retrouver un lieu purifié de l'attitude naturelle qui possède son vécu propre et ses modes propres d'intuition (il faudra discuter ce terme). La démarche phénoménologique (et transcendantale) implique une telle purification, ce qui appelle un arrachement difficile à la vie sociale en tant qu'elle est le niveau le plus ordinaire des vécus.

Cet article ne souhaite pas remettre en cause pour lui-même ce contrat phénoménologique. Il prétend en revanche aborder l'expression "appel de la conscience» à partir de deux sortes de thématiques: I) sociales, voire sociologiques, en un sens, puisqu'il s'agit de fonder l'usage des choses, qui est le premier rapport que nous avons au monde, dans une normativité sociale qui prescrit leur utilité aux choses: de ce point de vue, cette normativité est extrinsèque à l'individu qui fait usage, elle est commune aux membres de la même communauté et il n'y a d'ailleurs d'usage qu'à condition qu'il y ait une telle normativité collective; 2) transcendantales, lorsque plus originairement que ce plan social, il y va d'une praxis personnelle atteinte au moyen d'une réduction phénoménologique qu'il faudra qualifier, où la conscience morale (Gewissen), parfois, contredit la loi sociale, et 
m'impose le devoir de suivre sa voix. Plan transcendantal, donc, dans la mesure où il est plus originaire que la normativité sociale et fondateur d'une normativité qui possibilise mon être. Le Gewissen, pour être moral, n'en est pas moins transcendantal, dans la filiation de la conscience chez le Husserl du tournant transcendantal, mais en vue d'une réforme pratique de la phénoménologie qui a marqué les premiers moments de sa genèse.

Aussi souhaitons-nous d'abord montrer comment, chez Max Scheler, le Gewissen joue un rôle essentiellement négatif, en s'exprimant par un «non!» originaire qui arrête l'action sociale du sujet en manifestant l'inadéquation des normes sociales au profit d'une normativité plus originaire, qui se trouve dans les états de choses, ou encore les «états de valeur ", le corrélat noématique de l'acte intentionnel de valeur. Dans le deuxième moment, on montre comment Heidegger hérite sur ce point de Scheler, tout en radicalisant son geste de "conversion" (et donc en s'en distinguant), puisque la normativité authentique n'est plus celle des états de choses, mais celle du Dasein lui-même, ce qui ne va pas sans poser des problèmes éthiques difficiles concernant le statut de cette normativité. En somme, il s'agit de développer les bases théoriques à partir desquelles il est possible de discuter l'exigence phénoménologique de purification des vécus, et singulièrement des vécus sociaux. Décrire, en définitive, la pratique renouvelée de la réduction phénoménologique métamorphosée en termes éthiques, et les présupposés méthodologico-phénoménologiques d'une telle pratique.

\section{Scheler et l'objectivité axiologique}

La pensée de Max Scheler est, dans son grand ouvrage Der Formalismus in der Ethik de I9I3, une pensée de la valeur par laquelle il reprend la conceptualité fondamentale des Recherches logiques en terrain strictement pratique, exclusive de la dimension théorique qui est une autre façon de se rapporter à des objets. L'intuition donatrice joue alors un rôle fondamental pour penser le rapport pratique au monde:

c'est précisément l'un des critères de la nature essentielle (Wesensnatur) de constituants donnés, que, lorsque nous tentons de les "observer", il apparaisse toujours qu'il faut bien que nous les ayons déjà intuitionnés pour orienter l'observation dans le sens souhaité et présupposé (dass wir ihn immer schon erschaut haben müssen, um der Beobachtung die gewünschte und vorausgesezte Richtung zu geben),

et une telle intuition est à chaque fois présupposée lorsque nous complexifions notre rapport à l'objet ${ }^{3}$. Cette intuition est déjà valorisée, tout en

3. Max Scheler, Der Formalismus in der Ethik und die materiale Wertethik. Neuer Versuch der Grundlegung eines ethischen Personalismus, Halle, Max Niemeyer, I9I 6, p. 45 ; trad. Maurice de Gandillac, Paris, Gallimard, I99I, p. 72 (nous modifions souvent la traduction sans le signaler à chaque fois). 
étant originaire, dans une transposition du principe des principes husserlien du plan théorique au plan des valeurs. Ce qui est au fondement de toute représentation pratique, si l'on peut dire, a déjà une valeur (mais cela ne vaut, pour Scheler, que pour les objets que nous pratiquons; il y a bien, à côté de cela, un monde d'objets théoriques). La question qui doit demeurer à l'esprit est le mode de constitution de ces valeurs, et leur source - nous verrons cela plus loin.

L'autre notion husserlienne qui joue un rôle fondamental est l'a priori matériel, là encore transposé sur le plan des valeurs. Scheler entend l'a priori matériel en termes de biens et de valeurs, ce qui donne par exemple telle analyse:

Excluons donc absolument de [la catégorie de contenu sensible] le son, la couleur, la qualité olfactive et la qualité gustative; mais faisons-y entrer la faim, la soif, la douleur, le bien-être, la fatigue, ainsi que toutes ces sensations vaguement localisées dans des organes déterminés et qu'on appelle "sensations organiques". Tels sont les patrons (Musterbilder) des "sensations" et pour ainsi dire des sensations qu'on "sent"

De prime abord, ce que nous sentons, ce sont des sensations, c'est-àdire des tendances qui ont affaire à des biens, à des choses d'usage. Devant un gâteau, j'ai faim ou je suis écœuré, à quelque degré que ce soit; un son provoque d'emblée chez moi de l'agrément ou de la douleur, comme tous les autres sens. Une sensation, à ce niveau tout à fait primaire, a d'emblée une valeur affective et d'usage, et met en jeu mon corps propre. Sur ce plan immanent, je n'ai pas affaire à du rouge, mais à une tendance, une direction dit encore Scheler, en rapport avec mon Leib. Une pure qualité sensible, cela n'existe pas.

Nous avons ici une première réponse à la question de la source de la normativité des biens: il faut que les choses dont je fais usage soient valorisées à partir de la structure objective elle-même, puisque la valeur d'un objet est intuitivement donnée. Il y a chez Scheler, de ce point de vue, un ontologisme axiologique très fort, où la connaissance des valeurs peut atteindre l'évidence à condition de viser le bon état de choses, et correctement. C'est bien d'une phénoménologie qu'il s'agit, où l'objet ou l'état de choses, pensés en termes de biens (valorisés), doivent être donnés et donc remplis dans un acte intuitif pour se manifester à la conscience. Cela implique, du côté du sujet (qui est aussi bien un agent), une disposition intentionnelle fondamentale qui tend vers ces états de choses: «[Les valeurs] surgissent dans un échange sentant et vivant avec le monde (im fühlenden, lebendigen Verkehr mit der Welt) [...], dans l'amour et dans la haine même, c'est-à-dire dans la ligne d'accomplissement de ces actes intentionnels (in der Linie des Vollzugs

4. Ibid., p. 54 . 
jener intentionalen Akte) $)^{5}$.» C'est bien dans l'acte intentionnel lui-même que l'a priori matériel (axiologique/éthique) est intuitionné, dans le mouvement de la vie où je suis dans un rapport amoureux ou haineux aux choses qui m'entourent. Mais alors, l'acte intentionnel est une tendance, l'intention est comprise au sens axiologique, toujours prise dans un vouloir qui tend vers tel ou tel type d'étant, selon la détermination correspondante de l'a priori matériel, toujours dépendant in fine du mode de donation des états de choses axiologiques. Cela n'implique pas qu'il y ait d'abord l'intuition d'un objet-valeur, puis la tendance préférentielle qui tend vers cet objet. Dans l'intuition de l'objet-valeur il y a déjà la tendance préférentielle, et la donation a lieu dans la préférence: "On ne peut pas dire que la supériorité d'une valeur soit ressentie, exactement comme l'est la valeur singulière, et qu'ensuite la valeur supérieure soit "préférée" ou "subordonnée". En réalité la supériorité d'une valeur n'est donnée selon la nécessité de son essence que dans l'acte préférentiel ${ }^{6}$.» Scheler reprend ici le couple acte intuitiflacte signitif des Recherches logiques, mais en termes axiologiques: le donné ressenti d'un objet/valeur n'est possible que pour un acte préférentiel qui donne son milieu au donné et peut l'accueillir. Il n'y a cependant d'essence axiologique (et il est d'ailleurs étonnant, nous le verrons, qu'il y en ait une!) qu'à condition que cette essence soit donnée, sans quoi il ne s'agirait plus de phénoménologie, fût-elle axiologique.

Qu'il s'agisse d'une reprise du principe des principes husserlien en termes phénoménologiques, tel passage l'atteste:

La hiérarchie des valeurs ne peut jamais être déduite. Quelle valeur est "supérieure", c'est toujours par l'acte de préférence et de subordination que nous devons le saisir de nouveau. Il existe à cet effet une "évidence préférentielle" intuitive que ne peut remplacer aucune déduction logique (es gibt hierfür eine intuitive 'Vorzugsevidenz', die durch keinerlei logische Deduktion zu ersetzen ist $)^{7}$.

Autrement dit, une intuition originaire de valeur remplit un acte de préférence, et non pas un acte intentionnel théorique.

\section{Scheler et la normativité contextuelle}

Or la structure conceptuelle des Recherches logiques ne suffit pas à l'entreprise de Scheler. En effet, et c'est sensible assez vite dans l'ouvrage de I9I6, la question s'impose du lieu originaire des vécus, la conscience où se constituent les actes intentionnels de valeur. Est-elle un «Je transcendantal»? Le «Je transcendantal» formel est universel, et par conséquent les valeurs qui

5. Der Formalismus in der Ethik..., op. cit., p. 64; trad. cit., p. 90.

6. Ibid., p. 85 ; trad. cit., p. I08: «Man darf nicht sagen, es werde das Höhersein eines Wertes genau so 'gefühlt' wie der einzelne Wert selbst, und es werde dann der höhere Wert sei es 'vorgezogen', sei es 'nachgesetzt'. Vielmehr ist das Höhersein eines Wertes wesensnotwendig nur im Vorziehen 'gegeben'. "

7. Ibid., p. 87-88; trad. cit., p. I I I. 
valent pour lui valent nécessairement, parce qu'elles sont à la fois formelles et a priori, pour tous les autres "Je" également conçus comme transcendantaux. Or, au nom d'un matérialisme phénoménologique, Scheler veut penser l'individualité de la "personne» qui accomplit les actes intentionnels de valeur, et par conséquent son rapport individuel à des valeurs qui par ailleurs peuvent être reconnues de façon identique par tous, précisément parce que la source normative semble se situer au plan des objets eux-mêmes. C'est alors que Scheler nomme pour la première fois la "conscience morale » (Gewissen), qui possède cette individualité. Sans cette conscience morale, nous n'observons que des "Je individuels» qui ne sont qu'empiriques, et nullement originaires, car commandés par un «Je transcendantal» faussement individuel, et véritablement universel, sans personnalité car sans individualité. Autrement dit, Scheler joue l'ego phénoménologique individuel voire personnel contre le «Je transcendantal» kantien universel et générique.

Cette conscience morale (Gewissen), qui est une anticipation de la «personne» comme concept le plus originaire pour qualifier l'individu pratique, possède une faculté anticipatrice de l'action, que Scheler nomme «éthique du succès » (Erfolgsethik):

Le phénomène du vouloir se réduit en réalité tout simplement à une tendance, dans laquelle un contenu est donné comme destiné à être réalisé (im Phänomen des Wollens überhaupt liegt zunächst nichts als dies, dass es ein Streben ist, in dem ein Inhalt als ein $z u$ realisierender gegeben ist). Par là le vouloir se distingue de toutes les simples "aspirations" (Ausstreben), mais aussi du "souhait" (Wünschen), lequel est une tendance (Streben), dont la visée intentionnelle (seiner Intention nach) n'a pas pour fin la réalisation de son contenu (nicht auf die Realisierung eines Inhaltes selbst abzielt $)^{8}$.

Le souhait n'engage pas l'action, il est d'une certaine manière en deçà de la praxis. Lorsqu'il y a véritablement "vouloir », c'est-à-dire praxis, alors il y va d'une téléologie, d'une pré-actualisation du possible que l'on vise. Cela implique que l'on ait l'expérience des actions, que l'on acquière une telle expérience par la répétition des actions. Un enfant, écrit Scheler, peut "vouloir» (et non pas seulement "souhaiter») "qu'une étoile lui tombe sur les genoux $»^{9}$. Il peut le vouloir le plus sérieusement du monde. L'expérience lui apprendra peut-être que ce n'est pas possible, et si cela arrive, l'expérience montrera encore que son vouloir n'y était en fait pas du tout impliqué. Par l'expérience des causes et des effets, nous intégrons au vouloir lui-même les fins qu'il se donne, ou plutôt qui lui sont données par les états de choses. Ainsi, l'expérience complexifie toujours plus les buts que l'on se donne, que l'on considère comme possibles. Mais ce que montre a contrario l'exemple de l'enfant, c'est que notre "vouloir» est primitivement un vouloir-de-

8. Ibid., p. I23; trad. cit., p. I43.

9. Ibid., p. I 23. Plus loin, il souligne que les rêves de l'enfance ne sont précisément pas, pour l'enfant, des rêves. Ils le deviennent. 
contenu, un vouloir toujours déjà matérialisé par un contenu préalable; c'est ensuite que, l'expérience décevant les attentes, certains buts ne peuvent plus qu'être souhaités, et non voulus. C'est en ce sens que la conscience est toujours en régime pratique "Gewissen» : elle est une volonté, non pas formelle, mais toujours déjà engagée dans la matérialité de l'action à venir, dans les états de choses qui me transforment en se donnant.

Cependant, sommes-nous toujours à des états de choses qui donnent leur valeur aux objets? Autrement dit, la valeur provient-elle toujours de la structure objective des états de choses, ou encore des a priori synthétiques matériels qui sont donnés, ou bien n'y a-t-il pas une autre provenance, moins "ontologique ", de la valeur? Scheler est bien conscient que, la plupart du temps, nous agissons non pas conformément aux états de choses, mais plutôt conformément à des normes sociales bien spécifiques. C'est ce que Scheler appelle le «Milieu», le contexte en somme qui donne de la valeur aux objets. À propos des choses de milieu, c'est-à-dire des objets pris en contexte, Scheler parle d'un «domaine intermédiaire entre notre contenu perceptif et les objets qui le constituent d'une part, et d'autre part les objets objectivement pensés (Zwischenreiche zwischen unserem Perzeptionsinhalt und seinen Gegenständen und jenen objektiven gedachten Gegenständen) ${ }^{10}$ ». Ce domaine intermédiaire signifie que la chose prise dans son milieu n'est pas conforme à l'état de choses objectif, mais en même temps qu'elle n'est pas le seul contenu perceptif dans la conscience. Il s'agit d'une source normative contextuelle:

Ainsi le "milieu» (Milieu) ne se réduit pas à la série des objets que je perçois quand je marche dans la rue ou quand je suis assis dans ma chambre (qu'il s'agisse de sensations ou de représentations), mais il comprend aussi tout ce dont je tiens pratiquement compte (ich praktisch rechne), en tant que cela est présent ou absent, identique ou différent, par exemple les voitures et les hommes que j'évite (perdu moi-même dans mes pensées ou alors que j'ai les yeux dirigés au loin sur un autre homme). De même le matelot peut « compter » sur une tempête prochaine à partir des variations de son "milieu ", sans pouvoir dire que telle modification déterminée (par exemple la formation de nuages, la température, etc.) lui sert de signe (Zeichen) pour cette prévision ${ }^{11}$.

Ainsi l'objet, en s'inscrivant dans un Milieu, acquiert-il une signification particulière en devenant un signe, un signe sans objet pour ainsi dire, puisque le bruit du moteur dans la rue ne renvoie pas à l'objet-voiture, mais à la nécessité pour moi de traverser rapidement, ou encore de ne pas traverser - il renvoie à l'action elle-même, et c'est en cela que c'est un signe sans objet. De même le matelot, voyant les nuages, ne voit pas l'objet-nuage avec ses caractéristiques, mais il voit immédiatement un signe pour une action, réduire la voilure, par exemple, pour éviter d'endommager la toile

10. Ibid.

11. Ibid., p. I4I ; trad. cit., p. I 58. 
ou les mâts. D'ailleurs, Scheler souligne bien que je n'ai pas besoin de voir la voiture ou le nuage; d'une certaine manière, c'est présent dans le contexte, je vois le tout, sans précisément porter mon regard sur l'objet. Le signe apparaît en contexte, quand je suis à ce contexte même, ce qui me permet de "tenir pratiquement compte» (expression frappante) des objets de ce contexte. Scheler ajoute, à ce propos:

Le praticien (der Praktiker) [...] est pour ainsi dire entouré d'unités chosales (umringt von dinghaften Einheiten), qui se présentent à lui indépendamment de la perception qu'il peut en avoir, comme un royaume d'actions efficaces présentant divers niveaux et qualitativement singularisées (als ein Reich abgestuster und qualitativ gesonderter Wirksamkeiten darstellen), royaume qui doit être déjà singularisé et articulé (schon gesondert und gegliedert) pour servir de point d'appui à une conduite possible; et l'homme d'action "apprend" à "faire le tour" de ces unités, sans avoir aucune sorte de savoir théorique concernant les lois qui les régissent ${ }^{12}$.

La hiérarchie des valeurs est ici gouvernée par le milieu qui donne aux objets leur mode de présence, sans visée intentionnelle d'un objet, mais dans une saisie de tout le contexte où les choses sont nivelées, mises en relief eu égard à leur valeur propre. Il en va sans doute, ici, d'une remise en question du paradigme husserlien du voir: je ne vois pas un signe, je ne vois pas davantage le contexte qui lui donne sa valeur, mais je ressens le tout affectivement et pratiquement — j'en «fais le tour".

En fait, si je ne perçois pas ces milieux de façon objective, c'est qu'ils dépendent de représentations sociales tout à fait identifiables - Scheler donne l'exemple de deux marcheurs dans la même forêt, l'un qui se promène, l'autre qui chasse. Dans les deux cas, le milieu n'est pas le même, et les choses de ce milieu ne sont pas les mêmes signes: l'oiseau qui s'envole sera la source d'un émerveillement dans un cas, et le signal pour la mise en joue dans l'autre. Il est tout à fait clair que l'appréhension des objets et plus largement de la valeur de ces objets provient pour une large part de représentations préalables sociales, les unes appartenant à la sphère de la "promenade pour se détendre ", les autres appartenant à la sphère de l'activité de la chasse. Ce que Scheler nomme "Sittengesetze", lois de mours propres à chacune de ces activités, dont la normativité est socialement (et non pas «objectivement », ou alors il s'agit d'un type social d'objectivité bien spécifique) déterminée.

12. Ibid., p. I4I-I42. Scheler souligne encore que c'est le milieu qui conditionne la perception (ibid., p. I50): "Mais en réalité les sensations (Empfindungen) ne sont données (gegeben), absolument parlant, à un être vivant qu'autant que (et dans les limites où) elles ont une fonction indicatrice pour les choses (im denen sie Zeigefunktion für Dinge haben), et de plus pour les choses de son milieu global (für Dinge seines Gesamtmilieus).» 


\section{Scheler et la réduction pratique: le rôle du Gewissen}

Max Scheler reconnaît donc l'opérativité de «Sittengesetze», lois de mœurs ou plus simplement lois sociales, pour les perceptions ordinaires et axiologiques d'objets. Mais comment une telle pensée de la normativité sociale pour l'intentionnalité est-elle compatible avec une pensée phénoménologique de la donation d'états de choses valorisés dans un certain type d'intuition (de ressenti) axiologique? En fait, cette difficulté est le lieu d'une tension, voire d'une opposition. Car si cette normativité sociale est inévitable, elle entre parfois en contradiction, de façon morale, avec la structure objective des valeurs. C'est alors au Gewissen de jouer son rôle, et donc, à une certaine pratique de la réduction phénoménologique:

Ce qui constitue l'essence même de la conscience morale (Gewissen), c'est le sédiment du discernement (Einsicht) que j'ai (à partir de ma propre expérience de vie (Lebenserfahrung) de ce qui est bon, en tant que cela est «bon pour moi»). En ce sens il appartient essentiellement à la conscience morale de ne pouvoir être remplacée par aucune "norme", aucune «loi de mœurs» (Sittengesetze), etc. Son action (Leistung) commence précisément là où cesse celle de ces normes et de ces lois, là où la conduite (Handeln) et le vouloir (Wollen) satisfont déjà à leurs exigences. Il en résulte que dans une situation identique, plus ma conscience parle purement (je reiner es redet), plus elle doit dire autre chose que ce que dit la conscience d'autrui, et elle se tromperait certainement si elle parlait de la même façon! A la "conscience» prise en ce sens on peut appliquer sans réserves le principe de liberté de conscience (Prinzip der Gewissensfreiheit), ce qui signifie que - dans les questions qui ne sont (et qui par nature ne sauraient être) résolues par la partie objectivement valable des principes axiologiques de contenu discernables (einsichtigen) et des normes bâties sur ces principes - , chacun est libre (frei) d'écouter la voix de sa conscience (seinem Gewissen Gehör zu geben). En face des prétentions injustifiées de lois de mœurs (Sittengesetze) n'ayant d'autre validité qu'universelle, ce principe assure donc la sauvegarde du droit de l'individu moral en tant qu'individu (das Recht des sittlichen Individuums als Individuum). C'est précisément pourquoi la conscience et la liberté de conscience (Gewissensfreiheit) ne portent atteinte ni à l'idée d'un bien objectif, dont la conscience est justement l'organe permettant de la connaître dans la mesure où il s'agit de ce qui est objectivement bon "pour» un individu, ni à l'idée et aux prérogatives d'un discernement (Einsicht) universellement valable concernant les principes de valeur et les normes pour tous (für Alle geltender Wersätze und Normen) ${ }^{13}$.

Scheler parle de "Sittengesetze», qui sont les lois en vigueur dans une communauté donnée, que ces lois soient positives ou simplement tacites. On remarque que la voix du Gewissen résonne avant ces lois, qu'elle n'en a pas besoin car elle est, en elle-même, un discernement, un regard dans... (Einsicht), qui porte sur des objet-valeurs - et il est bien clair que la «liberté de 
conscience» demeure une conscience en vue d'objets, en vue de valeurs objectives. Cette Einsicht est pure ( $j$ je reiner es redet...»), c'est-à-dire pure de toute normativité sociale. Tout en étant objective, cette connaissance intuitive (Einsicht) n'est pas sociale, communautaire, elle ne parle pas comme parle la conscience d'autrui, elle est donc souverainement individuelle: c'est à l'individu en tant qu'individu, dit encore Scheler, qu'elle parle, et elle donne donc ainsi ce qui est bon "pour moi ». C'est-à-dire, plus précisément, qu'elle est parfaitement adaptée à l'individu qui l'entend, tout en demeurant aux objets absolument valides, aux essences de valeurs qui sont dans les objets. Une loi communautaire injuste, qui semble à l'Einsicht du Gewissen injuste, est une loi qui est de facto dépassée par la voix de la conscience, entièrement libre car entièrement individuelle. Alors, la voix de la conscience ne parle pas le langage du commerce quotidien et social, et elle est le lieu de la liberté (Freiheit). Cette purification phénoménologique de la subjectivité sociale en subjectivité pour ainsi dire conscientielle est bien une réduction phénoménologique où la norme de l'appréciation des valeurs passe du plan social (Sittengesetze) au plan de la conscience dont la voix seule permet d'orienter vers l'essence objective des valeurs, par-delà donc la normativité sociale. La réduction phénoménologique est aussi bien pratique qu'éthique: elle conduit au Gewissen dont la voix oriente vers les véritables valeurs des états de choses telles qu'elles sont données.

Mais l'automaticité de nos pratiques sociales, l'agilité de notre rapport aux normes sociales ne rend-elle pas impossible une telle réduction phénoménologique purifiant les vécus de ces normes? Comment briser la voix de la «majorité », pour reprendre le mot de Scheler ${ }^{14}$ ? Il est clair que le Gewissen est une faculté de regard dans..., d'Einsicht, de discernement de l'objetvaleur. Le Gewissen est donc dépendant d'un objet qui n'est pas lui. On peut ne pas écouter sa voix, écrit Scheler ${ }^{15}$, sans que cela remette en cause une seconde l'objet-valeur comme tel. Cependant, le Gewissen ne s'exprime pas positivement. Il ne produit donc aucun jugement axiologique comme tel (même s'il les fonde). Voici comment Scheler détaille le mode d'expression du Gewissen:

Ajoutons à cela que, prise en son sens propre, la conscience morale (Gewissen) fonctionne essentiellement de façon négative (wesentlich negativ funktioniert). Elle se présente quelque chose comme mauvais, comme ne devant-pas-être, elle "élève des protestations", etc. (es stellt als schlecht dar, als nichtseinsollend, es «erhebt Einspruch» usw.). Lorsque nous disons: «la conscience se dresse...» (das Gewissen regt sich...), cela signifie immédiatement qu'elle s'oppose d'une certaine façon à tel comportement (Verhalten), non pas qu'elle dise jamais que quelque chose est bon (nie aber: das Gewissen sagte, es sei etwas gut). C'est pourquoi la "mauvaise conscience» (schlechte Gewissen) est

14. Ibid., p. 338 .

15. Ibid., p. 333 . 
un phénomène beaucoup plus nettement positif que la «bonne conscience» (eine entschieden positivere Erscheinung wie das "gute Gewissen»), laquelle se réduit, en face d'un comportement déterminé qui pose un problème moral, à l'absence vécue de toute "mauvaise conscience». Même avant une décision de vouloir, lorsqu'on «consulte sa conscience », la conscience "met en garde» et «défend» bien plutôt qu'elle ne recommande et n'ordonne (auch vor einer Willensentscheidung, wenn man mit seinem "Gewissen zu Rate geht", «warnt» und "verbietet» das Gewissen mehr, als es empfiehlt oder gebietet). Sa fonction n'est pas de fournir un discernement originairement positif, mais de critiquer, tantôt en mettant en garde, tantôt en condamnant (so hat es keine ursprünglich positive Einsicht gebende, sondern $n u$ reine kritische, teils warnende, teils richtende Funktion $)^{16}$.

Ce qui conseille ou déconseille, c'est la "voix de la conscience" (Stimme des Gewissens), pour reprendre l'expression de Scheler, qui est ou non entendue correctement, ou qui peut tout simplement mal juger d'un état de valeurs. Ce Gewissen appelle d'une façon particulière, originairement sur le mode de la mauvaise conscience; elle a ainsi une fonction "essentiellement négative». Ce qu'elle fait le plus souvent, c'est qu'elle "interpelle», elle "élève des objections", ou mieux, elle "fait appel»: "es erhebt Einspruch ${ }^{17}$. Ce "faire appel» (au sens judiciaire) interpelle le sujet dans sa conduite, le Gewissen met en garde, et ne désigne rien de bon. Sa positivité se manifeste dans une interpellation essentiellement négative, puisque la conscience est originairement mauvaise conscience ${ }^{18}$. En ce sens, elle n'est pas originaire, mais elle ne peut prétendre qu'à être une faculté très importante de discernement, loin donc d'être le lieu de la "voix de Dieu", loin donc d'être infaillible. En ce sens, elle est un lieu subjectif qui a affaire à de l'objectif: elle est la faculté de discernement, donc, de ce qui est bon en tant que c'est bon pour moi, à partir de mon expérience de vie (Lebenserfahrung). Elle est la faculté qui permet de substituer aux lois sociales, issues des traditions ou de la loi positive, son appréciation des valeurs, et en ce sens elle est un lieu pour la liberté individuelle, puisque "chacun est libre d'écouter sa conscience ${ }^{19} »$. Cette liberté joue un rôle capital, car elle montre que les

16. Ibid., p. 334 .

17. Ibid., p. 334 .

18. Ibid. Citons ce passage plus largement: «Dazu tritt, dass das Gewissen - seinem Wortsinne nach - wesentlich negativ funktioniert. Es stellt als schlecht dar, als nicht seinsollend, es 'erhebt Einspruch' usw. Sagen wir: 'das Gewissen regt sich', so bedeutet dies ohne weiteres soviel wie: Es wehrt sich etwas gegen das betreffende Verhalten, nie aber: das Gewissen sagte, es sei etwas gut. Darum ist auch das 'schlechte Gewissen' eine entschieden positivere Erscheinung wie das 'gute Gewissen', das für ein bestimmtes, sittlich in Frage gestelltes Verhalten eigentlich nur das erlebte Fehlen und der erlebte Mangel des 'schlechten Gewissens' ist. Auch vor einer Willensentscheidung, wenn man mit seinem 'Gewissen zu Rate geht', 'warnt' une 'verbietet' das Gewissen mehr, als es empfiehlt oder gebietet. So hat es keine ursprünglich positive Einsicht gebende, sondern nu reine kritische, teils warnende, teils richtende Funktion. " 19. Ibid., p. 337 . 
valeurs objectives sont intangibles, que la conscience interpelle le sujet lorsqu'une norme sociale contrevient à ce qu'elle discerne (et donc juge, si l'on comprend bien Scheler) de l'objet-valeur. Cette interpellation exhibe la différence qu'il y a alors entre ce que la norme sociale dicte, et ce que l'objetvaleur incite à faire. L'appel de la conscience nous extrait, lorsqu'il le faut (et ce n'est pas tout le temps le cas) de la normativité sociale, et nous met négativement (par un avertissement, par la mauvaise-conscience) en face de l'objectualité des valeurs en jeu dans telle action. Cela implique donc une différence tout aussi radicale entre la conscience (Gewissen), et la norme, c'est-à-dire l'objectivité-valeur. Le Gewissen, pour Scheler, est toujours dépendant d'une objectivité axiologique. C'est la raison pour laquelle elle est indépendante vis-à-vis de toute normativité collective: elle est la "voix» qui interpelle, c'est-à-dire qui alerte lorsque la norme que l'on suit contrevient à l'état de choses/valeurs.

On voit alors comment la conscience phénoménologique se trouve métamorphosée en termes éthiques, et comment un topos théologique, philosophique et littéraire comme la «voix de la conscience» permet à Scheler d'éthiciser la phénoménologie à partir du problème des valeurs et de la normativité qu'elles impliquent. Plus encore, on voit comment un tel appel "purifie» les vécus sociaux en vécus souverainement individuels, ou plus exactement en vécus libres où l'individu est directement aux prises avec les états de choses axiologiques, avec le donné axiologique. La normativé objective, seule vraiment valable, s'oppose parfois à la normativité sociale, et il revient alors au sujet d'entendre la mise en garde de sa conscience morale. Cela pourrait susciter une rupture du contrat social; mais Scheler est prudent, et souligne que la conscience morale ne dit rien de positif. En disant simplement «non », elle laisse la volonté du sujet libre de comprendre cette mise en garde, mais aussi libre de ne pas la comprendre, ou encore de l'adapter aux circonstances. Néanmoins, il en va ici d'une éthique de la franchise, et (en un sens non technique) de l'authenticité, voire de la transparence. Il s'agit bien d'un anti-constructivisme phénoménologique fondamental pour lequel, si la normativité sociale joue un rôle indéniable dans l'ensemble de nos rapports aux valeurs, elle doit toujours reposer sur une appréciation à la première personne des états de choses tels qu'ils sont donnés. Le Formalisme en éthique permet donc de reconnaître l'existence et l'importance de la normativité sociale tout en la subordonnant à un réalisme phénoménologique où le donné, indépendamment de cette normativité, joue le rôle cardinal. C'est une réduction phénoménologique pratique, voire éthique, l'appel de la conscience, qui permet de purifier les vécus afin d'atteindre le plan originaire et pur du donné axiologique.

\section{Heidegger et le «on» comme norme}

Sans revenir sur les analyses de la mondanéité du monde et sur l'ustensilité dans Être et temps, on notera la grande proximité de la pensée de l'usage 
chez Heidegger avec la pensée des «signes » chez Scheler. Pour Heidegger, les étants sont autant d'outils, c'est-à-dire de "signes" en vue d'usages déterminés, dans un contexte, une "tournure» systématique, un système de renvois où chaque signe prend son sens par rapport à d'autres signes, ce qui était implicitement pensé avec le concept de «milieu» chez Scheler. Pour reprendre l'exemple de Scheler, lorsque je marche dans la rue, les objets sont autour de moi comme des outils, c'est-à-dire qu'ils sont pris en considération dans un certain usage conformément au contexte de cet usage, à savoir le fait que je marche dans la rue pour me rendre par exemple à l'université. La voiture n'est pas un objet-voiture, mais elle est d'emblée la voiture qui m'empêche de traverser, l'eau qui ruisselle le long du caniveau est d'emblée perçue comme cette eau que je dois enjamber pour monter sur le trottoir, etc. Un outil est précisément cette structure systématique où les objets sont toujours perçus en vue de quelque chose d'autre qu'eux, comme signe renvoyant à la totalité de la tournure qui est ce à quoi je suis pour parvenir aux fins de mon action. Ce n'est pas le lieu ici de détailler une telle conception, mais nous y reviendrons à propos d'un détail important de l'analyse heideggérienne de l'appel de la conscience, dans la deuxième section d'Être et temps.

Nous voudrions approfondir, avec Heidegger, l'importance d'une réduction phénoménologique éthique du rapport contextuel aux choses sur le plan individuel d'un sujet en définitive pratique, qui doit se conformer à une normativité d'un tout autre type que la normativité quotidienne et sociale. L'enjeu est pour nous de montrer comment, dans le même sens fondamental que Scheler, Heidegger pratique également une réduction éthique, tout en questionnant la différence non moins fondamentale entre les deux, touchant notamment au statut du donné et de la normativité, et donc - en définitive - de la phénoménologie ${ }^{20}$.

20. L'héritage de Scheler, si important soit-il, doit cependant, du point de vue de l'histoire conceptuelle, être relativisé, dans la mesure où si en maint endroit Heidegger reconnaît sa dette, il indique aussi les différences fondamentales. Soit GA 20, p. I74-I75 (trad. Alain Boutot, Paris, Gallimard, 2006, p. I87-I 88): «La voie suivie par Scheler pour déterminer l'intentionnel et les actes, la personne et l'homme, ne va pas fondamentalement plus loin dans la mesure où Scheler est également axé sur la définition traditionnelle de l'homme comme animal rationale ", même s'il "se détourne, pour déterminer les vécus, les actes et le Je, de l'orientation relevant de la raison théorique qui est celle de Husserl». Si l'on comprend bien, l'importance de Scheler est d'avoir orienté la phénoménologie hors des sentiers théoriques pour penser les actes intentionnels de façon pratique, en terme de personne et d'effectuation. Cependant, il est demeuré prisonnier du vocabulaire à la fois des Recherches logiques et des Ideen I de Husserl, ce qui inscrit le projet même du Formalisme en éthique dans une contradiction indépassable. En d'autres termes, "tout ce qui est dit [par Scheler], c'est ceci: des actes sont effectués et la personne est l'effectuateur des actes. Rien n'est dit sur le mode d'être de l'effectuation des actes et le mode d'être de leur effectuateur" (ibid., p. I77; trad. cit., p. I90). Il a manqué à Scheler une analytique existentiale qui remonte plus loin dans les conditions de possibilité des actes intentionnels, même si "Scheler est bien celui qui, sous l'influence de Bergson, est allé 
Voici, au $\$ 55$ d'Être et temps, la façon dont Heidegger présente le rôle de l'appel de la conscience:

En se perdant dans la publicité du "on » et son bavardage, il mésentend, n'entendant que le «on" -même, son Soi-même propre, Si le Dasein doit pouvoir être ramené - et certes par lui-même - hors de cette perte de la mésentente de soi, alors il faut qu'il puisse d'abord se trouver, lui-même qui s'est mésentendu et mésentend dans l'écoute du «on». Cette écoute doit être brisée, autrement dit il faut que lui soit donnée par le Dasein même la possibilité d'un entendre qui l'interrompe. La possibilité d'une telle rupture se trouve dans l'être-appelé immédiat. L'appel brise l'écoute prêtée au «on» par un Dasein qui se mésentend, lorsque, conformément à son caractère d'appel, il éveille un entendre qui est en tous points caractérisé de manière opposée à l'entendre perdu. Si celui-ci est pris par le "vacarme» de la multiple équivoque d'un bavardage chaque jour "nouveau ", il faut que l'appel appelle sans vacarme, sans équivoque, sans point d'appui pour la curiosité. Ce qui donne à comprendre en appelant ainsi, c'est la conscience ${ }^{21}$.

Ce que décrit désormais Heidegger, c'est l'attestation du "pouvoir-être authentique» du Dasein, ce qui suppose qu'il s'était jusqu'alors perdu. Pourquoi s'était-il perdu? parce qu'il n'était pas lui-même, mais il était ce qu' "on» lui prescrit d'être. Pour définir ce que Heidegger appelle «on » ici, et son corollaire, la "publicité » (Öffentlichkeit), il faut revenir sur ce qu'il entend par le verbe "hören", puisque le "on" est ici défini à partir de l'écoute. Retournons au $\$ 34$ d'Être et temps: l'«entendre» (Hören) est un phénomène associé au "comprendre»: "Das Dasein hört, weil es vers$t e h t^{22}$.» Pour que j'écoute, il faut être en contexte, c'est-à-dire être dans une attitude compréhensive, même si par exemple je n'ai pas suivi la conversation. Il n'y a d'écoute qu'en contexte. Et si je ne connais pas le contexte, je

aujourd'hui le plus loin dans cette question» (ibid.). Nous montrons dans notre interprétation de Heidegger les différences fondamentales (et phénoménologiques) entre les deux pratiques de la réduction phénoménologique qui ont cependant en commun tout à la fois l'extraction hors des pratiques socialement normées et un certain rôle joué par l'appel de la conscience, même s'il n'a assurément pas les mêmes caractéristiques. Nous remercions un des lecteurs anonymes pour la suggestion de cette critique de Scheler par Heidegger.

21. Ibid., p. 27I : «Sich verlierend in die Öffentlichkeit des Man und sein Gerede überhört es im Hören auf das Man-selbst das eigene Selbst. Wenn das Dasein aus dieser Verlorenheit des Sichüberhörens soll zurückgebracht werden können - und zwar durch es selbst — dann muß es sich erst finden können, sich selbst, das sich überhört hat und überhört im Hinhören auf das Man. Dieses Hinhören muß gebrochen, das heißt es muß ihm vom Dasein selbst die Möglichkeit eines Hörens gegeben werden, das jenes unterbricht. Die Möglichkeit eines solchen Bruchs liegt im unvermittelten Angerufenwerden. Der Ruf bricht das sich überhörende Hinhören des Daseins auf das Man, wenn er, seinem Rufcharakter entsprechend, ein Hören weckt, das in allem gegenteilig charakterisiert ist im Verhältnis zum verlorenen Hören. Wenn dieses benommen ist vom 'Lärm' der mannigfaltigen Zweideutigkeit des alltäglich 'neuen' Geredes, muß der Ruf lärmlos, unzweideutig, ohne Anhalt für die Neugier rufen. Was dergestalt rufend zu verstehen gibt, ist das Gewissen.»

22. Ibid., p. I63. 
le reconstruis déjà à l'avance: je m'approche d'un groupe de collègues, je les entends parler sans distinguer les mots. Peu à peu j'entends les mots - mais toujours déjà précède le contexte: je sais à quoi m'attendre, puisque ce sont des collègues, et qu'entre collègues, on parle souvent des mêmes choses. Toute écoute est fondée sur une telle compréhension du contexte où s'inscrit chaque son comme dans un système. C'est pourquoi "De prime abord" (Zunächst), nous n'entendons jamais des bruits et des complexes sonores, mais toujours la voiture qui grince ou la motocyclette. Ce qu'on entend, c'est la colonne en marche, le vent du nord, le pivert qui frappe, le feu qui crépite $^{23}$.» Autrement dit, ce que j'entends est toujours un outil, en-main (zuhanden), puisque je fais usage de ce que j'entends, je l'inscris dans un contexte de compréhension, et c'est ainsi que les mots d'une phrase prennent un sens. Ce que disent mes collègues, par exemple, a du sens dans l'horizon du contexte universitaire, où chaque phrase et chaque mot trouvera sa signification non pas d'abord théorique, mais pratique: il s'agit de ce que nous ferons après le colloque, de la fatigue qui nous prend après avoir parlé, etc.

Dans le cours de 1925 sur le "concept de temps », Heidegger est plus précis:

Prêter l'oreille, c'est aussi écouter en comprenant, ce qui signifie qu'on ne perçoit pas "originairement et de prime abord" des bruits ou des complexes sonores, mais la voiture qui grince, le "tram", la motocyclette, la colonne en marche, le vent du nord. Il faut se placer dans une attitude très artificielle et pour le moins sophistiquée pour pouvoir "se mettre à l'écoute" d'un "bruit pur" ${ }^{24}$.

Et Sein und Zeit, derechef, précise:

Mais que nous entendions de prime abord des motocyclettes et des voitures, c'est la preuve phénoménale que le Dasein en tant qu'être-au-monde séjourne à chaque fois déjà auprès de l'en-main (Zudanden) intramondain, et non pas d'abord auprès de "sensations" (Empfindungen) dont le "fouillis" (Gewühl) devrait être préalablement mis en forme (geformt) pour confectionner le tremplin permettant au sujet (Subjeckt) d'atteindre enfin un "monde" 25 .

23. Ibid.: «'Zunächst' hören wir nie und nimmer Geräusche und Lautkomplexe, sondern den knarrenden Wagen, das Motorrad. Man hört die Kolonne aud dem Marsch, den Nordwind, den klopfenden Specht, das knisternde Feuer. "

24. GA 20, p. 367 ; trad. cit., p. 385 : «Auch das Horchen ist verstehendes Hören, d. h. ursprünglich und zunächst hört man gerade nicht Geräusche und Lautkomplexe, sondern den knarrenden Wagen, die Elektrische das Motorrad, die Kolonne auf dem Marsch, den Nordwind.»

25. Sein und Zeit, op. cit., p. I64: «Dass wir aber zunächst Motorräder und Wagen hören, ist der phänomenale Beleg dafür, dass das Dasein als In-der-Welt-sein je schon beim innerweltlich Zuhandenen sich aufhält und zunächst gar nicht bei 'Empfindungen', deren Gewühl zuerst geformt werden müsste, um das Sprungbrett abzugeben, von dem das Subjeckt abspringt, um schliesslich zu einer 'Welt' zu gelangen. » Cf. sur ce dossier le précieux commentaire de Dominique Pradelle, "De Husserl à Heidegger: intentionnalité, monde et sens ", dans Discipline filosofiche, $\mathrm{XXV}, \mathrm{n}^{\circ}$ 2, 20I 5, p. 46: «Ce qui demeure valable, c'est la destitution du 
Nulle réduction (et nulle distinction d'ailleurs entre sensibilité et entendement, entre un pur donné intuitif et une mise en forme par l'entendement, à la façon néokantienne), mais une analyse existentiale des conditions de donation de sens, et son premier principe: l'herméneutique, le rapport compréhensif/intoné au monde. Le Hören auf... est précisément le concept pour penser une telle écoute d'étants bien compris - il est «auprès» de l'étant qu'il entend, c'est-à-dire l'étant en-main, zuhanden. Plus encore, l'écoute est d'emblée communautaire parce que compréhensive: en écoutant je suis à la communauté, je pratique un ensemble commun de signes, au sein de jeux de langage socialement spécifiques. On voit pourquoi, dans le texte du $\$ 55$ que nous commentons ici, Heidegger parlait de la "publicité du "on" » (Öffentlichkeit des Man) en termes d' «écoute» (Hören): ce qu'on écoute, ce sont des signes qui fonctionnent exactement comme les outils que l'on pratique. La plupart du temps, c'est le son du moteur de la voiture qui m'indique que je ne dois pas traverser, avant que je voie cette voiture. La «vue» n'a plus le privilège au sein de cette réforme pratique de l'intentionnalité husserlienne.

Il faut donc comprendre que les outils que j'entends, si l'on peut parler ainsi, prennent leur valeur à partir de normes sociales, "publiques ", qui rendent possible la communauté. "On» ne traverse pas lorsque le feu est rouge, "on» utilise la fourchette de telle façon, "on" utilise telles formules pour se parler (selon le contexte d'énonciation), etc. Les outils sont des outils pour un "on ", ce qui coupe le Dasein de son être authentique, au sens où il perd alors son identité, son individualité: il est disséminé dans le "on ", il est comme tous les autres, il n'est plus une "personne » à part entière. Son action n'obéit qu'aux normes collectives, et tout est outil pour lui. Ici se manifeste une première et profonde différence avec Scheler: Heidegger, nulle part, ne parle d'un donné d'états de choses que seraient les outils ou encore leur contexte de tournure. L'approche de Heidegger est bien plutôt herméneutique, où il n'y a pas de place pour un donné intuitif qui précèderait ce contexte, la systématique des signes et sa pratique. Il y a un enchevêtrement du Dasein compréhensif et de la systématique où il se trouve, un "être-aumonde" toujours déjà auprès des signes qui sont des outils au sein de la pratique qu'il en fait. La normativité sociale est tout à fait capitale ici,

privilège méthodologique de la réflexion transcendantale comme donation adéquate des vécus: le Dasein — ou le soi qui lui est propre — n'est pas donné par la réflexion transcendantale qui dévoilerait les vécus immanents; en effet, puisqu'il se transcende lui-même et se trouve primairement auprès des choses dont il se préoccupe, il se comprend en fonction de ce auprès de quoi il s'affaire, c'est-à-dire à partir des choses, de l'étant intra-mondain. [...] Ainsi, d'une part, cette phénoménologie ne thématise plus guère de sensations ou de contenus hylétiques: on n'y sait plus rien de sensations, de données hylétiques ressenties (les sensations acoustiques et moments de couleur éprouvés), mais on y perçoit d'emblée des qualités sensibles (le son du violon ou de la voiture qui passe, la couleur de ce mur ou du soleil couchant). Le Dasein étant d'emblée êtreauprès-de..., le sensible relève de ce auprès de quoi il se trouve, et non de quelque sphère de vécus internes et ressentis.» 
puisque toutes les analyses herméneutiques de Heidegger sont reconductibles en dernière instance au "on » qui gouverne les pratiques: le «on" est bien collectif, et en tant qu'il est intrinsèquement lié à l' «Öffentlichkeit», il est social.

Mais si Heidegger refuse explicitement, dans le texte que nous citions à l'instant, une réduction théorique qui reconduirait les vécus intentionnels de valeur à une intuitivité d'objet originaire, il promeut néanmoins, avec l'appel de la conscience, une réduction éthique, qui reconduit le Dasein depuis la dissémination de son être jusqu'à son être authentique. Cette réduction vise à briser l'écoute du "on ", dit le texte du $\$ 55$, c'est-à-dire à détourner le Dasein de l'usage des outils pour le placer devant lui-même. Qu'est-ce à dire, et comment la conscience pourrait-elle effectuer cette réduction éthique?

\section{Heidegger et l'appel comme réduction éthique}

Heidegger écrit: «L'appel n'énonce rien, il ne donne aucune information sur des événements du monde, il n'a rien à raconter (der Ruf sagt nichts aus, gibt keine Auskunft über Weltereignisse, hat nichts zu erzählen ${ }^{26}$." Dans une proximité frappante avec Scheler, Heidegger rend effectif l'appel de la conscience par l'indétermination de son message, par la négativité fondamentale de son contenu. Si l'appel était entendu sur le mode des énoncés habituels, s'il résidait dans des phrases que je prononcerais à moi-même, alors ce mode d'énonciation ne diffèrerait pas, quant à l'essence, du mode quotidien d'expressivité. Mais Heidegger radicalise en fait Scheler: plus encore que la négativité, c'est le "faire-silence " (Schweigen) qui caractérise l'appel, le Schweigen que le $\mathbb{3 4}$ du traité analysait amplement. La conscience appelle en silence, mais «ne perd rien en perceptibilité (Vernehmlichkeit) ${ }^{27}$ ». Il y a donc un Vernehmen qui saisit le silence de l'appel. Il s'agit d'une perception, d'une saisie, Vernehmen, d'un devoir: celui de se détourner du «on" brusquement interrompu, interruption qui laisse résonner une autre possibilité d'existence, non affairée. On voit bien, ici, que la radicalisation heideggérienne de Scheler reste proche de Scheler: car qu'est-ce que le silence, sinon ce qui est le contraire du bavardage du " on " quotidien, c'està-dire un faire-silence qui rompt singulièrement avec la " tonalité ambiante ", pour ainsi dire?

Cependant, cela ne nous dit pas d'où vient la nouvelle normativité qui prétend se substituer à la normativité sociale propre au «on». Des états de choses, états de valeur, à l'instar de Scheler? non pas:

L'appel, précisément, n'est pas et n'est jamais ni projeté, ni préparé, ni accompli volontairement par nous-mêmes. "Ça" appelle, contre notre attente, voire

26. Sein und Zeit, op. cit., p. $273\left(\$_{56} 6\right)$.

27. Ibid. 
contre notre gré (der Ruf wird ja gerade nicht und nie von uns selbst weder geplant, noch vorbereitet, noch willentlich vollzogen. 'Es' ruft, wider Erwarten und gar wider Willen) ${ }^{28}$.

La norme est donnée dans le Vernehmen par un «ça ", qui semble constituer une garantie de normativité, parce que le Dasein n'est pas, dans la réduction éthique opérée grâce à l'appel de la conscience, sa propre norme (que voudrait dire une normativité qui s'adresse à moi et dont je serais l'instigateur ?). "Es», c'est "ça », un indéterminé d'où provient l'appel et qui rompt le bavardage, "ça » qui ne provient pas du vouloir, mais s'adresse à lui. La différence avec Scheler est ici massive, et engage la teneur phénoménologique de l'analyse: la purification du plan du «on" à un plan plus originaire ne dévoile pas la primauté normative des états de choses donnés à la conscience. Il me remet bien plutôt à moi-même, au nom selon nous d'un subjectivisme pratique (bien éloigné donc du réalisme phénoménologique de Scheler) où le Dasein rompt avec ses pratiques quotidiennes en s'appelant soi-même.

Cependant, Heidegger précise que ce n'est pas «moi» qui appelle ${ }^{29}$. Est-ce du même coup prétendre que l'appel ne provient pas du Dasein? Non pas, car:

[L'appelant] est le Dasein en son étrang(èr)eté (Unheimlichkeit), il est l'être-aumonde originellement jeté en tant qu'hors-de-chez-lui (Un-zuhause). Il est le "que" nu (nackte 'Dass') dans le rien du monde (Nichts der Welt). L'appelant est non familier au "on" - même quotidien - quelque chose comme une voix étrangère (eine fremde Stimme). Et qu'est-ce qui pourrait être plus étranger (fremder) au "on", perdu qu'il est dans la diversité du "monde" de sa préoccupation, que le Soi-même isolé sur soi dans l'étrang(èr)eté, jeté dans le rien ${ }^{30}$ ?

Au $\$ 40$, Heidegger anticipait sur ces pages, où l'angoisse brisait déjà la familiarité quotidienne, et était décrite déjà la modalité du «hors de chez soi », précisément l'étrang(èr)eté qui est reprise dans ces pages sur l'appel ${ }^{31}$. C'est une instance plus fondamentale au Dasein quotidien qui appelle, c'està-dire très précisément une possibilité insigne de son existence: la possibilité de ne plus pratiquer des outils, la possibilité de l'authenticité, d'une existence recueillie auprès de Soi-même. Le fondement est cette possibilité même. Il n'est donc rien de fixe, mais la tendance vers une autre existence,

28. Sein und Zeit, op. cit., p. 275.

29. Ibid., p. 276.

30. Ibid., p. 276-277.

31. Ibid., p. I89: "La familiarité quotidienne se brise. Le Dasein est isolé, mais comme être-au-monde. L'être-à revêt la "modalité" existentiale du hors-de-chez-soi. Ce n'est pas autre chose que veut dire l'expression "d'étrang(èr)eté" (das Dasein ist vereinzelt, das jedoch als Inder-Welt-sein. Das In-sein kommt in den existenzialen 'Modus' des Unzuhause. Nichts anderes meint die Rede von der 'Unheimlichkeit').» Sur l'angoisse comme réduction, voir Jean-François Courtine, Heidegger et la phénoménologie, op. cit. 
le pouvoir-être soi-même du Dasein - ce que nous nous risquons à appeler, pour bien marquer la différence avec le réalisme schelérien tout autant que pour identifier l'orientation fondamentale d'Etre et temps, un subjectivisme pratique.

Du même coup, la normativité est fondée dans et par ce qui permet la réduction éthique même, ce qui ne sonne absolument pas communément, une tonalité éthique, une interpellation qui me place devant le pur factum de l'existence, et qui m'isole en moi-même en me «jetant» dans cet isolement, dans le rien — rien à faire, rien à produire, rien à pratiquer, rien à modifier, rien à attendre, rien à..., mais également le rien plus fondamental que la mort annonce sur ce mode du projet. La réduction est du même coup pleinement transcendantale, puisqu'il n'y a plus de séparation entre le processus de la réduction et son résultat: dès lors qu'il y a réduction, il y aussitôt son effet, le Soi-même, l'identité du Dasein, cette identité qui appelle par delà le «on», en passant par lui, en le brisant. Le transcendantal est un fait et une possibilité tout à la fois. Le fait des faits, si l'on peut dire, un fait sans ce qu'il y a à faire, où le faiseur est en train de se faire, et où il est seul à faire - plus précisément un "avoir-à-faire », de et par soi-même.

En somme, l'appel traque, poursuit au sens quasi policier: "Diese Unheimlichkeit setzt dem Dasein ständig nach und bedroht, wenngleich unausdrücklich, seine alltägliche Verlorenheit in das Man", phrase du $\$ 40$ d'Être et temps qu'on retrouve quasiment à l'identique au $\$ 57$ : "die Unheimlichkeit setzt dem Dasein nach und bedroht seine selbstvergessene Verlorenheit» — «nachsetzen» voulant dire suivre, traquer aussi. L'Unheimlichkeit est l'expressivité même de l'appel, la menace qui pèse sur toute praxis ordinaire des outils. Alors qu'il semble que tout y est, que tout s'offre ou se refuse à nous, que l'expérience est bien celle d'un tout inévitable, une tonalité inquiétante se fait entendre. Là est la radicalisation heideggérienne de la négativité du Gewissen chez Scheler : la voix de la conscience, ne disant rien, me projette vers le rien, et donne l'injonction d'une rupture avec les normes sociales où «on» agit ${ }^{32}$. Avec la réduction éthique est gagnée la "Vereinzelung", l'individuation du Dasein qui, dans la dissémination des outils du monde, était lui-même disséminé dans l'ensemble de la communauté, dans

32. Il y aurait lieu de rapporter l'Unheimlichkeit à deux sources, au moins: la schellingienne, "was im Geheimnis, im Verbogenen, in der Latenz, bleiben sollte und hervorgetreten ist ", qui joue évidemment un grand rôle pour Heidegger; mais également la freudienne, qui fait fond sur Schelling, par exemple dans Das Unheimliche (I9I9), où l'Unheimlichkeit est justement associée à l'Angst, sans parler du rôle que Freud fait en général jouer au "Es ", ni de celui du concept de "Schuld». Sur les liens possibles entre Heidegger et Freud, voir Havi Cavel, Life and Death in Freud and Heidegger, Amsterdam/New York, Rodopi, 2006. Voir aussi D. F. Krell, "Schlag der Liebe, Schlag des Todes. On a Theme in Heidegger and Trakl», dans John Sallis (ed.), Radical Phenomenology, New Jersey, Humanities Press, 1978, p. 238-258, ou du même, "Das Unheimliche: Architectural Sections of Heidegger and Freud ", dans Research in Phenomenology, $\mathrm{n}^{\circ}$ I7, I992, p. 43-6I. 
le « on" qui est autant un tel qu'un autre. L'Unheimlichkeit est ici, à la source de l'appel, "Unheimlichkeit der geworfenen Vereinzelung ${ }^{33}$ ", étrang(èr)eté de l'individuation jetée. Chez Scheler, déjà, le Gewissen était exclusivement l'affaire de l'Individuum insoumis et libre. Au sens courant, la conscience morale ne s'adresse qu'à moi seul, elle ne se partage pas, elle ne s'adresse pas à plusieurs sujets. Elle est ce qui est proprement mien. Heidegger reprend aussi cela à Scheler: sa voix est exclusivement mienne, et en retentissant elle me singularise, m'individualise. C'est donc par l'Unheimlichkeit issue de l'appel de la conscience que le Dasein atteint l'individu de son Soi-même propre, qui n'est rien d'autre que jeté, projeté, c'est-à-dire dans la facticité même de son existence où $i l$ y a à être. C'est en ce sens précis qu'on peut parler de subjectivisme pratique.

Quant à l'explication avec Scheler, elle est d'ailleurs implicite à la fin $\mathrm{du} \$ 57$, où on lit: "Comment la conscience pourrait-elle fonctionner comme con-vocatrice au pouvoir-être le plus propre alors que, de prime abord et le plus souvent, elle se borne à réprimander et à avertir (Wie soll das Gewissen als Aufrufer zum eigensten Seinkönnen fungieren, wo es doch zunächst und zumeist nur rügt und warnt)?" Scheler faisait bien du Gewissen une instance qui avertit $(w a r n t)^{34}$, et il s'agit pour Heidegger de se demander comment peut bien avertir la conscience, sur quel mode - ou, en d'autres termes, comment la négativité pourrait trouver une effectivité, et donc une positivité. Là où Scheler décrivait le Gewissen en termes de "mauvaise conscience» («mauvaise conscience» qu'Être et temps récuse, comme concept, au $\$ 59$, avec beaucoup de force, parce qu'il manquerait la dimension d'appel en moralisant un concept d'abord et avant tout ontologique - mais sur ce point, il n'est pas du tout certain que l'ontologie heideggérienne ne soit pas précisément à rapprocher d'une certaine morale, fût-elle par-delà ou avant bien et mal), Heidegger fait intervenir le concept de «Schuld», qu'E. Martineau traduit par «dette», mot qui nous semble bien convenir parce qu'il colore tout autant l'appel qu'il contient déjà une action latente du Dasein, une action qu'il faudra de toute façon accomplir: une dette est «à honorer", elle fait toujours signe vers l'avenir, vers l'à-faire. Cette dette me contraint d'affronter le devoir d'être moi-même, et alors commence véritablement, dans Être et temps, l'attestation d'une l'authenticité définitivement libérée de la normativité sociale, et donc enfin libre.

Nous souhaitons conclure de deux façons. Tout d'abord en rappelant la dimension phénoménologique des deux approches interprétées; ensuite, en posant des questions critiques à l'analyse heideggérienne, précisément à partir de sa dimension phénoménologique.

I) Ce qui frappe, chez Scheler, c'est l'importance proprement phénoménologique accordée aux états de choses axiologiques, qui demeurent

33. Être et temps, op. cit., p. 280.

34. Der Formalismus in der Ethik..., op. cit., p. 338. 
l'horizon normatif par excellence de toute pratique. Les valeurs sont inscrites dans ces états de choses, et la réduction éthique opérée depuis l'appel de la conscience ne fait au fond qu'exhiber, en purifiant les vécus, la source objective de toute normativité axiologique. De ce point de vue, il y a bien un primat phénoménologique du donné intuitif (Scheler parle d'«évidence intuitive »), métamorphosé en ressenti (car on ne "voit " pas à proprement parler les signes que l'on pratique), et par conséquent un primat du donné sur le construit. La réduction éthique montre la supériorité axiologique de la valeur inscrite dans l'état de choses tel qu'il se donne par rapport aux valeurs construites socialement (Sittengesetze). Chez Heidegger, c'est différent: il n'est plus question d'un donné des outils que l'on pratique, mais c'est au sein de la pratique même que l'outil est outil, dans une corrélation plus intime encore entre Dasein et choses. Cependant, à l'instar de Scheler, une réduction éthique, par le moyen de l'appel de la conscience, vient purifier la teneur sociale des pratiques quotidiennes, pour reconduire le Dasein à lui-même, pris par le choix qu'il a toujours à faire d'exister authentiquement, en deçà de l'Öffentlichkeit. La réduction éthique réduit la dissémination du Dasein dans le "on" et l'individualise radicalement. Là est sans doute le maintien du contrat phénoménologique chez Heidegger, dans cette purification de l'attitude naturelle par une réduction phénoménologique, certes métamorphosée en réduction éthique, mais néanmoins reconduisant la conscience (entendue alors comme Gewissen) à ses premières possibilités d'existence, possibilité pré-sociales.

2) Mais alors, les apories sont nombreuses. Tout d'abord, que pourrait bien être un individu qui ne "bavarde» pas? un individu qui ne se consacrerait, du matin jusqu'au soir, qu'à l'activité philosophique ou encore à la méditation silencieuse, au risque constant de se perdre en réintégrant l'espace social, l'Öffentlichkeit, ne serait-ce qu'un instant? Il y a assurément une piste moins austère pour comprendre le sens du propos de Heidegger. L'appel de la conscience trouverait sa norme dans l'espace public lui-même, et il s'agirait alors pour le Dasein de prendre notamment conscience de la dimension sociale de son être, et de l'aliénation qu'elle implique. Voyons ces deux points l'un après l'autre.

a) La norme est dans l'Öffentlichkeit elle-même. Heidegger insiste à de nombreuses reprises sur le fait que ce qui brise l'écoute du "on » est le contraire du «on », le "silence». Mais qu'est-ce que le silence, sinon le revers (et seulement cela) du bavardage du "on"? Il ne peut y avoir de silence véritable, c'est-à-dire de brèche dans le "on », précisément que dans le "on », à même le "on", qui décide alors du mode d'énonciation de ce qui le brise, qui est en d'autres termes la norme de son opposé, ou la contre-norme. Autrement dit, Heidegger prend appui sur le mode quotidien d'être au monde, qui est un mode social, pour parvenir jusqu'à son opposé, mode associable qui me rapproche plus purement de mon être dans sa constitution transcendantale. 
b) Du même coup, ce qui est en jeu avec l'appel de la conscience est non pas tant l'exclusion de la sphère sociale qu'une prise de conscience de cette sphère, ou encore une objectivation (pour utiliser là encore un vocabulaire fort peu heideggérien). Par la réduction éthique, l'individu parvient à poser la question du sens de l'être, c'est-à-dire à prendre conscience de son mode d'être au monde, tel qu'il est décrit dans Être et temps (qui de ce point de vue déploie un type de discours performatif, qui fait ce qu'il décrit). Il ne pratique plus le marteau, mais il voit tout ce qui est en jeu, de façon transcendantale (c'est-à-dire comme condition de possibilité) pour pratiquer le marteau: quelle structure du monde, quelle constitution du Dasein, les existentiaux ici à l'œuvre, et plus largement les concepts fondamentaux de l'analytique existentiale, jusqu'à la mort elle-même. Si Être et temps a pour ambition de nous extraire de la normativité sociale, c'est pour décrire ses conditions de possibilité, ce qui exige un écart avec la praxis, une normativité philosophique qui procède d'autres exigences que celles de l'usage quotidien. L'exigence et donc la normativité philosophique est d'un autre ordre, elle est transcendantale, mais elle manifeste du même coup la façon dont nous ne sommes pas tout à fait nous-mêmes quand nous sommes aux outils, la façon dont nous nous oublions la plupart du temps dans l'usage, tout à la tâche que nous sommes en train d'accomplir. Cependant, qu'est-ce qui légitime la validité de l'appel de la conscience que décrit Heidegger? Où l'authenticité trouve-t-elle sa norme? La praxis philosophique ne procède-t-elle pas elle-même d'une normativité sociale ? l'université, le cadre académique en général, ou encore une normativité de l'exercice philosophique tributaire des usages traditionnels, des pratiques antécédentes? De ce point de vue, il y aurait lieu d'inscrire plus en profondeur la normativité sociale, peut-être même sur le plan transcendantal, ce qui aurait peut-être pour effet tout autant de prolonger les résultats heideggériens que de les contester. 